\title{
Clinical Experience in the Treatment of Vulvovaginal Candida Disease with Integrated Traditional Chinese and Western Medicine
}

\author{
Xiaodan Yang1 ${ }^{1}$ Jie Wang1, Zhenzhen Jia ${ }^{1}$, Lihong Zhu' ${ }^{2 *}$ \\ ${ }^{1}$ Shaanxi University of Traditional Chinese Medicine, Xianyang 712000, Shaanxi Province, China \\ 2The Second Affiliated Hospital of Shaanxi University of Traditional Chinese Medicine, Xianyang 712000, Shaanxi Province, \\ China \\ *Corresponding author: Lihong Zhu, 1712799596@qq.com

\begin{abstract}
Summarizing two cases of vaginal candidiasis, cured by integrated traditional Chinese and western medicine in clinical work, it is found that the combination of traditional Chinese and western medicine has a significant effect on the treatment of this disease. The curative effect is outstanding, and the recurrence rate is significantly reduced, which can be used as a reference for gynecological medical workers.
\end{abstract}

Keywords: Integrated traditional Chinese and western medicine; Vulvovaginal candidiasis; Syndrome differentiation and treatment

Publication date: July 2021; Online publication: July 31, 2021

\section{Introduction}

The cause of vaginal candidiasis is clear. It is mainly caused by Candida albicans infection. The main symptoms are damp vulva with varying degrees of itching and increased vaginal discharge. At present, western medicine treatment is first to eliminate the main cause of the disease, and to apply antibacterial drugs locally or systemically. The main treatment of traditional Chinese medicine (TCM) is dehumidification, dialectical treatment according to different syndrome types, and at the same time cooperate with appropriate external treatment methods. Integrated Chinese and Western medicine can complement each other's advantages and improve clinical efficacy.

\section{Western medicine pathogenesis}

The pathogenic mechanism of Candida albicans is mainly because it can adhere to the vaginal stratified squamous epithelial cells. The vaginal epithelium has more folds, and the vaginal wall is rich in venous clusters. Candida albicans is easy to invade the vagina. Epithelium, and Candida has the ability to deform. When the body's resistance is reduced, the adhesion of Candida albicans will increase, form hyphae, produce proteases and other substances to increase its toxicity, and use its own deformation. Ability to invade the body and cause inflammation.

\section{TCM etiology and pathogenesis}

Vulvovaginal candida disease belongs to the category of "carrying disease" in TCM according to its clinical diagnosis. "Dampness disease" is caused by damp pathogens, and the dysfunction of the spleen and stomach is the internal condition of the onset. The disease is located in the anterior yin and uterus. Renal vein damage 
and loss of the vein are related to the pathogenesis. Dampness is in the progress of the disease. It can be damp heat from yang to heat, or cold to damp from yin ${ }^{[1]}$. The main syndrome types are spleen deficiency and dampness syndrome, kidney yin deficiency and damp-heat syndrome, damp-heat betting syndrome, dampness accumulation syndrome. The following are two cases of vulvovaginal candidiasis that I have observed in my clinical work.

\section{Clinical cases}

\subsection{Case 1}

Patient Jin, female, married, 24 years old, came to the hospital for diagnosis and treatment mainly because of "vulvar pruritus for ten days and aggravation for three days." The patient developed vulvar pruritus ten days ago and increased vaginal discharge. Three days ago, vulvar pruritus worsened, sitting sleeping restlessly, self-report of sticky and uncomfortable mouth, recent poor appetite, sleepy, irritable and irritable, sticky and uncomfortable stools, tenesmus, yellowish and thick yellowish color, short red urine. Checked the body and found that patient had red tongue, yellow greasy coating, slippery pulse. Gynecological examination showed, micro-erosion of the vulva, hyperemia of the vaginal mucosa, and tofu-like secretions in the vagina, which had a foul smell. The vaginal secretions were taken and submitted for examination and reported positive for Candida. Western medicine diagnosis was vulvovaginal candidiasis. TCM diagnosis was to take too much, damp and hot betting type. In treatment, western medicine gave oral itraconazole capsules. TCM provides TCM lotion for vulvar fumigation and washing. TCM composition, Sophora flavescens $15 \mathrm{~g}$, wild chrysanthemum $12 \mathrm{~g}$, white fresh bark $10 \mathrm{~g}$, Coptis $5 \mathrm{~g}$, Kochia scoparia $8 \mathrm{~g}$, Hedyotis diffusa 8g, Phellodendron amurense 10g, Scutellaria baicalensis 15g, Cnidium 5g, Cortex Moutan 12g, Nepeta $10 \mathrm{~g}$, Forsythia $10 \mathrm{~g}$, etc., were treated continuously for 14 days. The examination of vaginal secretions was negative when the patient returned. There was no recurrence during the half-year follow-up period. Itraconazole is a synthetic azole antifungal drug with a triazole ring. Its antibacterial spectrum is similar to fluconazole, and it has antibacterial effects on deep and superficial fungi ${ }^{[2]}$. It can inhibit Candida albicans in the vaginal epithelium. It is combined with Sophora flavescens to clear heat and dampness, kills insects and relieve itching, Phellodendron amurense to clear heat and dry dampness, wild chrysanthemum to clear heat and detoxify, and to cool blood and remove blood stasis from Moutan cortex.

\subsection{Case 2}

Patient Zhang Mou, female, married, 33 years old, three months postpartum, the main cause was "vulvar pruritus, pain for half a month, aggravated with increased vaginal discharge for three days," and he developed vulvar pruritus and pain with increased vaginal discharge for half a month. Self-administered After taking nystatin for ten days, the symptoms were slightly relieved, and the drug was stopped by itself. Three days ago, the symptoms of vulvar itching were aggravated, and curdled leucorrhea appeared. He complained of pain in the lower abdomen, irritability, short red urine, yellow stool, dry stool difficulty, red tongue, yellow greasy coating, slippery pulse. Gynecological examination was congestion of the vaginal mucosa, tofu-like secretions, foul smelling and unpleasant smell. Take the vaginal secretion and submit it for test report, Candida positive. Western medicine diagnosis was vulvovaginal candidiasis. Diagnosis by TCM was too much dampness and poison accumulation. Treatment with miconazole suppository + Wuwei disinfection drink, continuous treatment for seven days. Routine examination of vaginal discharge in the clinic after the next menstruation, Candida is negative and the patient is cured. There was no recurrence during the half-year follow-up. The patient's postpartum cell veins are empty, careless about health, the infection of damp toxin directly invades the vaginal organs and uterus, and the damp toxin damages the two veins, resulting in the loss of the veins and the loss of appointment ${ }^{[2]}$. Miconazole suppository is an imidazole chemical. It is the basic medicine for clinical treatment of vulvovaginal candida disease. Its 
efficacy and safety have been verified. Its mechanism of action is to inhibit the synthesis of fungal cell membrane and affect its metabolic process. Wuwei disinfecting drink can remove evil toxins invading and vaginally, in addition to damp toxins that damage any two veins ${ }^{[3]}$, and achieve the purpose of treating this disease together.

\section{Clinical experience}

From the comparison between the treatment of the above two cases and the previous empirical treatment, the treatment of vaginal candidiasis with combination of TCM and western medicine is better than the treatment of western medicine or TCM alone. The simple application of antibiotics in the treatment of vaginal candidiasis takes effect quickly, but it is easy to produce drug resistance, the treatment is incomplete, and the symptoms are easy to recur. However, the TCM treatment takes a long time to take effect, but the effect is stable and the side effects are small. Combination of TCM and Western medicine to treat this disease, antibiotics can be used to inhibit the growth of Candida, and pure western medicine cannot completely eliminate the symptoms and the shortcomings are easy to repeat. Significantly improve the patient's symptoms, improve the efficacy, and enhance the quality of life of the patient. The combined application of the two can not only effectively kill pathogenic bacteria, but also significantly improve symptoms and achieve the purpose of cure. In general, the combined treatment of vulvovaginal candidiasis with TCM and western medicine is better than simple TCM or western medicine, and it is worthy of clinical application.

\section{Funding}

Reproductive and Nutritional Metabolism Innovation Team（303/132041934）

\section{Disclosure statement}

The author declares no conflict of interest.

\section{References}

[1] 2013, "The Patient's Postpartum Veins were Empty, the Patient's Health Care was Careless, the Evil of Damp Toxins Directly Invaded the Vaginal Organs and Uterus, and the Damp Toxins Damaged Either the Two Veins, Resulting in the Loss of the Veins," 11(29) :389-390.

[2] Chen Y, 2014, Observation on the Efficacy of Itraconazole Combined with Fluconazole in the Treatment of Recurrent Candida Vaginitis. Medical Aesthetics, 15(8): 326-327.

[3] Hu C, Jian H, Chen J, et al., 2020, Clinical Observation on the Treatment of Sequelae of Pelvic Inflammatory Disease of Damp-Heat Stasis Syndrome by Oral Administration of Qingre Tiaoxue Decoction combined with Wuwei Disinfection Decoction. Chinese Journal of Experimental Pharmacology, 20:111-116. 